\title{
A Note on Laplace Transforms of Some Common Distributions Used in Counting Processes Analysis
}

\author{
Eugene Kouassi ${ }^{*}$, Edouard Akpata ${ }^{2}$, Koffi Pokou ${ }^{3}$ \\ ${ }^{1}$ Department of Economics, University of the Western Cape, Cape Town, South Africa \\ ${ }^{2}$ Department of Mathematics, University Felix Houphouet Boigny, Abidjan, Cote d'Ivoire \\ ${ }^{3}$ Department of Economics, University Felix Houphouet Boigny, Abidjan, Cote d'Ivoire \\ Email: ^Ekouassi@uwc.ac.za
}

How to cite this paper: Kouassi, E., Akpata, E. and Pokou, K. (2020) A Note on Laplace Transforms of Some Common Distributions Used in Counting Processes Analysis. Applied Mathematics, 11, 67-75. https://doi.org/10.4236/am.2020.112007

Received: July 16, 2018

Accepted: January 18, 2020

Published: January 21, 2020

Copyright ( 2020 by author(s) and Scientific Research Publishing Inc. This work is licensed under the Creative Commons Attribution International License (CC BY 4.0).

http://creativecommons.org/licenses/by/4.0/

\begin{abstract}
An important problem of actuarial risk management is the calculation of the probability of ruin. Using probability theory and the definition of the Laplace transform one obtains expressions, in the classical risk model, for survival probabilities in a finite time horizon. Then explicit solutions are found with the inversion of the double Laplace transform; using algebra, the Laplace complex inversion formula and Matlab, for the exponential claim amount distribution.
\end{abstract}

\section{Keywords}

Laplace Transforms, Common Distributions, Analysis, Probability

Distributions, Counting Processes

\section{Introduction}

This paper seeks to derive Laplace transforms of common distributions used in counting processes analysis. In mathematics and with many applications in physics and engineering and throughout the sciences, the Laplace transform is a widely used integral transform. Denoted by $L\{f(t)\}$, it is a linear operator of a function $f(t)$ with a real argument $t(t \geq 0)$ that transforms it to a function $F(s)$ with a complex argument $s$. This transformation is essentially bijective for the majority of practical uses; the respective pairs of $f(t)$ and $F(s)$ are matched in tables. The Laplace transform has the useful property that many relationships and operations over the originals $f(t)$ correspond to simpler relationships and operations over the images $F(s)$. 
The Laplace transform is related to the Fourier transform, but whereas the Fourier transform expresses a function or signal as a series of modes of vibration (frequencies), the Laplace transform resolves a function into its moments. Like the Fourier transform, the Laplace transform is used for solving differential and integral equations. In physics and engineering it is used for analysis of linear time-invariant systems such as electrical circuits, harmonic oscillators, optical devices, and mechanical systems. In general, the Laplace transform is often interpreted as a transformation from the time-domain, in which inputs and outputs are functions of time, to the frequency-domain, where the same inputs and outputs are functions of complex angular frequency, in radians per unit time. Given a simple mathematical or functional description of an input or output to a system, the Laplace transform provides an alternative functional description that often simplifies the process of analyzing the behavior of the system, or in synthesizing a new system based on a set of specifications.

This paper is concerned with finding formulae for finite time survival probabilities, by the complex inversion of the corresponding Laplace transforms, following and expanding [1] [2] [3] [4] and [5].

By definition the Laplace transform of a distribution of density function $f$ defined on $\mathbb{R}$ or on a part of $\mathbb{R}$ is the function defined on the set $\mathbb{C}$ of complex numbers by (e.g. see [4] [5] [6] and [7]),

$$
F(z)=\int_{0}^{\infty} f(t) \mathrm{e}^{-z t} \mathrm{~d} t
$$

The rest of the paper proceeds as follows: Section 2 is related to the main results derived in the paper. Section 3 concludes the paper.

\section{Main Results}

Positive distributions are considered (e.g. see [6]-[11]).

\subsection{Exponential Distribution and Its Laplace Transform}

Proposition 1. The Laplace transform of the exponential distribution, $f(x)=\lambda \mathrm{e}^{-\lambda x}$, is

$$
F(z)=\frac{\lambda}{\lambda+z}, \quad \forall z / z \neq-\lambda
$$

\section{Proof of Proposition 1:}

The Laplace transform of the law of $X$ is defined as,

$$
F(z)=\int_{0}^{\infty} \lambda \mathrm{e}^{-\lambda t} \mathrm{e}^{-z t} \mathrm{~d} t \text {. Hence, } F(z)=\int_{0}^{\infty} \lambda \mathrm{e}^{-(\lambda+z) t} \mathrm{~d} t=\frac{\lambda}{\lambda+z}, \text { with } z \neq-\lambda .
$$

\subsection{Weibull Distribution and Its Laplace Transform}

Proposition 2. The Laplace transform of the Weibull distribution with parameters $\lambda, \alpha$ and density function $f_{X}(x)=\alpha \lambda x^{\alpha-1} \exp (-\lambda x \alpha)$ is 


$$
F(z)=\frac{\alpha \lambda \beta \Gamma(\alpha)}{(\lambda \alpha+z)^{\alpha}}
$$

with $z \neq-\lambda \alpha$ and $\Gamma$ is defined as, $\Gamma(\alpha)=\int_{0}^{\infty} x^{\alpha-1} \mathrm{e}^{-x} \mathrm{~d} x$.

\section{Proof of Proposition 2:}

The Laplace transform is by definition, $F(z)=\int_{0}^{\infty} f(t) \mathrm{e}^{-z t} \mathrm{~d} t$. Since,

$F(z)=\int_{0}^{\infty} \alpha \lambda t^{\alpha-1} \mathrm{e}^{-\lambda t \alpha} \mathrm{e}^{-z t} \mathrm{~d} t=\alpha \lambda \int_{0}^{\infty} t^{\alpha-1} \mathrm{e}^{-(\lambda \alpha+z) t} \mathrm{~d} t$. Replacing $z$ by $z+\lambda \alpha$, one gets, $\int_{0}^{\infty} t^{\alpha-1} \mathrm{e}^{-(z+\alpha \lambda) t} \mathrm{~d} t=\frac{\Gamma(\alpha)}{(\alpha \lambda+z)^{\alpha}}$. Thus, $F(z)=\alpha \lambda \int_{0}^{\infty} t^{\alpha-1} \mathrm{e}^{-(\lambda \alpha+z) t} \mathrm{~d} t$, that is $F(z)=\alpha \lambda \frac{\Gamma(\alpha)}{(\lambda \alpha+z)^{\alpha}}$. Hence, $F(z)=\frac{\alpha \lambda \Gamma(\alpha)}{(\lambda \alpha+z)^{\alpha}}$, with $z \neq-\lambda \alpha$.

\subsection{Normal Distribution and Its Laplace Transform}

Proposition 3. The Laplace transform of the normal distribution with known parameters $\mu$ and $\sigma^{2}$, and with a density function given by

$$
\begin{aligned}
f(x)=\frac{1}{\sigma \sqrt{2 \pi}} \mathrm{e}^{-\frac{(x-\mu)^{2}}{2 \sigma^{2}}}, \text { is } \\
F(z)=\mathrm{e}^{-\frac{\left(\mu+\sigma^{2} z\right)^{2}-\mu^{2}}{2 \sigma^{2}}}\left(1-\Phi\left(\frac{-\mu-\sigma^{2} z}{\sigma}\right)\right), \forall z \in \mathbb{C}
\end{aligned}
$$

where $\Phi$ is the cumulative distribution of the standard normal distribution.

Proof of Proposition 3:

The Laplace transform is by definition,

$$
\begin{aligned}
& F(z)=\int_{0}^{\infty} \frac{1}{\sigma \sqrt{2 \pi}} \mathrm{e}^{-\frac{(t-\mu)^{2}}{2 \sigma^{2}}} \mathrm{e}^{-z t} \mathrm{~d} t=\int_{0}^{\infty} \frac{1}{\sigma \sqrt{2 \pi}} \mathrm{e}^{-\frac{(t-\mu)^{2}}{2 \sigma^{2}}-z t} \mathrm{~d} t=\int_{0}^{\infty} \frac{1}{\sigma \sqrt{2 \pi}} \mathrm{e}^{-\frac{(t-\mu)^{2}+2 \sigma^{2} z t}{2 \sigma^{2}}} \mathrm{~d} t \\
& F(z)=\int_{0}^{\infty} \frac{1}{\sigma \sqrt{2 \pi}} \mathrm{e}^{-\frac{t^{2}-2\left(\mu-\sigma^{2} z\right) t+\mu^{2}}{2 \sigma^{2}}} \mathrm{~d} t \text {. Thus, } \\
& F(z)=\int_{0}^{\infty} \frac{1}{\sigma \sqrt{2 \pi}} \mathrm{e}^{-\frac{\left(t-\mu+\sigma^{2} z\right)^{2}-\left(\mu+\sigma^{2} z\right)^{2}+\mu^{2}}{2 \sigma^{2}}} \mathrm{~d} t=\mathrm{e}^{-\frac{\left(\mu+\sigma^{2} z\right)^{2}-\mu^{2}}{2 \sigma^{2}}} \int_{0}^{\infty} \frac{1}{\sigma \sqrt{2 \pi}} \mathrm{e}^{-\frac{\left(t-\mu-\sigma^{2} z\right)^{2}}{2 \sigma^{2}}} \mathrm{~d} t
\end{aligned}
$$

Using the change of variable, $y=\frac{t-\mu-\sigma^{2} z}{\sigma}$, one gets

$$
\begin{aligned}
& F(z)=\mathrm{e}^{-\frac{\left(\mu+\sigma^{2} z\right)^{2}-\mu^{2}}{2 \sigma^{2}}} \int_{-\frac{-\sigma^{2} z}{\sigma}}^{+\infty} \frac{1}{\sqrt{2 \pi}} \mathrm{e}^{-\frac{y^{2}}{2}} \mathrm{~d} y . \text { Therefore, } \\
& F(z)=\mathrm{e}^{-\frac{\left(\mu+\sigma^{2} z\right)^{2}-\mu^{2}}{2 \sigma^{2}}}\left(1-\int_{-\infty}^{\frac{-\mu-\sigma^{2} z}{\sigma}} \frac{1}{\sqrt{2 \pi}} \mathrm{e}^{-\frac{y^{2}}{2}} \mathrm{~d} y\right) .
\end{aligned}
$$


Hence, $F(z)=\mathrm{e}^{-\frac{\left(\mu+\sigma^{2} z\right)^{2}-\mu^{2}}{2 \sigma^{2}}}\left(1-\Phi\left(\frac{-\mu-\sigma^{2} z}{\sigma}\right)\right), \forall z$ complex, and where $\Phi$ is the cumulative density function of the standard normal distribution.

\subsection{Inverse Gaussian and Its Laplace Transform}

Proposition 4. The Laplace transform of the Inverse Gaussian distribution with parameters $\lambda, \mu$ and with a density function defined by

$$
\begin{gathered}
f(x)=\left(\frac{\lambda}{2 \pi x^{3}}\right)^{\frac{1}{2}} \exp \left(-\frac{\lambda(x-\mu)^{2}}{2 \mu^{2} x}\right), \text { is given by, } \\
F(z)=\frac{\left(\lambda+2 \mu^{2} z\right)^{\frac{1}{2}}}{\lambda^{\frac{1}{2}}} \mathrm{e}^{\frac{2 \lambda \mu^{4} z}{\left(\lambda+2 \mu^{2} z\right)^{2}}}
\end{gathered}
$$

where $z$ is a complex number such that: $\lambda+2 \mu^{2} z$ is a real positive number (e.g. see [7] [8] [9] and [10]).

\section{Proof of Proposition 4:}

By definition, $F(z)=\int_{0}^{\infty} f(t) \mathrm{e}^{-z t} \mathrm{~d} t$.

Since, $F(z)=\int_{0}^{\infty}\left(\frac{\lambda}{2 \pi t^{3}}\right)^{\frac{1}{2}} \exp \left(-\frac{\lambda(t-\mu)^{2}}{2 \mu^{2} t}\right) \mathrm{e}^{-z t} \mathrm{~d} t$, one gets,

$$
\begin{gathered}
F(z)=\int_{0}^{\infty}\left(\frac{\lambda}{2 \pi t^{3}}\right)^{\frac{1}{2}} \exp \left(-\frac{\lambda(t-\mu)^{2}}{2 \mu^{2} t}-z t\right) \mathrm{d} t \\
F(z)=\int_{0}^{\infty}\left(\frac{\lambda}{2 \pi t^{3}}\right)^{\frac{1}{2}} \exp \left(-\frac{\lambda(t-\mu)^{2}-2 \mu^{2} z t^{2}}{2 \mu^{2} t}\right) \mathrm{d} t
\end{gathered}
$$

Or, $F(z)=\int_{0}^{\infty}\left(\frac{\lambda}{2 \pi t^{3}}\right)^{\frac{1}{2}} \exp \left(-\frac{t^{2}\left(\lambda+2 \mu^{2} z\right)-2 \lambda \mu t+\lambda \mu^{2}}{2 \mu^{2} t}\right) \mathrm{d} t$

$$
\begin{gathered}
F(z)=\int_{0}^{\infty}\left(\frac{\lambda}{2 \pi t^{3}}\right)^{\frac{1}{2}} \exp \left(-\frac{\left(\lambda+2 \mu^{2} z\right)\left(t^{2}-\frac{2 \lambda \mu}{\lambda+2 \mu^{2} z} t+\frac{\lambda \mu^{2}}{\lambda+2 \mu^{2} z}\right)}{2 \mu^{2} t}\right) \mathrm{d} t \\
F(z)=\int_{0}^{\infty}\left(\frac{\lambda}{2 \pi t^{3}}\right)^{\frac{1}{2}} \exp \left(-\frac{\left(\lambda+2 \mu^{2} z\right)\left(\left(t-\frac{\lambda \mu}{\lambda+2 \mu^{2} z}\right)^{2}-\frac{\lambda^{2} \mu^{2}}{\left(\lambda+2 \mu^{2} z\right)^{2}}+\frac{\lambda \mu^{2}}{\lambda+2 \mu^{2} z}\right)}{2 \mu^{2} t}\right) \mathrm{d} t \\
F(z)=\mathrm{e}^{\frac{2 \lambda \mu^{4} z}{\left(\lambda+2 \mu^{2} z\right)^{2}} \int_{0}^{\infty}\left(\frac{\lambda}{2 \pi t^{3}}\right)^{\frac{1}{2}} \exp \left(-\frac{\lambda \mu}{\left(\lambda+2 \mu^{2} z\right)\left(t-\frac{\lambda \mu^{2}}{\lambda+2 \mu^{2} z}\right)}\right)} \mathrm{d} t
\end{gathered}
$$




$$
\begin{gathered}
F(z)=\mathrm{e}^{\frac{2 \lambda \mu^{4} z}{\left(\lambda+2 \mu^{2} z\right)^{2}}} \int_{0}^{\infty}\left(\frac{\lambda}{2 \pi t^{3}}\right)^{\frac{1}{2}} \exp \left(-\frac{\left(\lambda+2 \mu^{2} z\right)\left(t-\frac{\lambda \mu}{\lambda+2 \mu^{2} z}\right)^{2}}{2 \mu^{2} t}\right) \mathrm{d} t \\
F(z)=\mathrm{e}^{\frac{2 \lambda \mu^{4} z}{\left(\lambda+2 \mu^{2} z\right)^{2}}} \int_{0}^{\infty}\left(\frac{\lambda}{2 \pi t^{3}}\right)^{\frac{1}{2}} \exp \left(-\frac{\frac{\lambda^{2}}{\lambda+2 \mu^{2} z}\left(t-\frac{\lambda \mu}{\lambda+2 \mu^{2} z}\right)^{2}}{\frac{2 \mu^{2} \lambda^{2}}{\left(\lambda+2 \mu^{2} z\right)^{2}} t}\right) \mathrm{d} t
\end{gathered}
$$

Therefore,

$$
\begin{aligned}
& F(z)= \\
& \mathrm{e}^{\frac{2 \lambda \mu^{4} z}{\left(\lambda+2 \mu^{2} z\right)^{2}}} \times \lambda^{\frac{1}{2}} \times \frac{1}{\left(\frac{\lambda^{2}}{\lambda+2 \mu^{2} z}\right)^{\frac{1}{2}}} \int_{0}^{\infty}\left(\frac{\frac{\lambda^{2}}{\lambda+2 \mu^{2} z}}{2 \pi t^{3}}\right)^{\frac{1}{2}} \exp \left(-\frac{\frac{\lambda^{2}}{\lambda+2 \mu^{2} z}\left(t-\frac{\lambda \mu}{\lambda+2 \mu^{2} z}\right)^{2}}{\frac{2 \mu^{2} \lambda^{2}}{\left(\lambda+2 \mu^{2} z\right)^{2}} t}\right) \mathrm{d} t
\end{aligned}
$$

Considering the complex number $z$ such that, $\lambda+2 \mu^{2} z$ is a real positive number, one gets the above equality, $F(z)=\mathrm{e}^{\frac{2 \lambda \mu^{4} z}{\left(\lambda+2 \mu^{2} z\right)^{2}}} \times \lambda^{\frac{1}{2}} \times \frac{1}{\left(\frac{\lambda^{2}}{\lambda+2 \mu^{2} z}\right)^{\frac{1}{2}}}$

$$
\text { Since } \int_{0}^{\infty}\left(\frac{\frac{\lambda^{2}}{\lambda+2 \mu^{2} z}}{2 \pi t^{3}}\right)^{\frac{1}{2}} \exp \left(-\frac{\frac{\lambda^{2}}{\lambda+2 \mu^{2} z}\left(t-\frac{\lambda \mu}{\lambda+2 \mu^{2} z}\right)^{2}}{\frac{2 \mu^{2} \lambda^{2}}{\left(\lambda+2 \mu^{2} z\right)^{2}} t}\right) d t=1 \quad \text { it is the }
$$

integral on the domain of the density function of a law with parameters $\left.\lambda+2 \mu^{2} z, \frac{\lambda \mu}{\lambda+2 \mu^{2} z}\right)$. Hence, $F(z)=\frac{\left(\lambda+2 \mu^{2} z\right)^{\frac{1}{2}}}{\lambda^{\frac{1}{2}}} \mathrm{e}^{\frac{2 \lambda \mu^{4} z}{\left(\lambda+2 \mu^{2} z\right)^{2}}}, \forall z$ a complex number such that: $\lambda+2 \mu^{2} z$ is a real positive number (e.g. see [11] [12] [13] and $[14])$.

\subsection{Gamma Distribution and Its Laplace Transform}

Proposition 5. The Laplace transform of a Gamma distribution with parameters $\lambda, \beta$ and with a density function given by, $f(x)=\frac{\lambda \beta x^{\beta-1} \mathrm{e}^{-\lambda x}}{\Gamma(\beta)}$; is:

$$
F(z)=\frac{\lambda \beta}{(\lambda+z)^{\beta}}, \forall z / z \neq-\lambda
$$

where $\Gamma$ is defined as before, that is $\Gamma(\alpha)=\int_{0}^{+\infty} x^{\alpha-1} \mathrm{e}^{-x} \mathrm{~d} x$.

\section{Proof of Proposition 5:}


As $F(z)=\int_{0}^{\infty} f(t) \mathrm{e}^{-z t} \mathrm{~d} t$; one can write

$$
F(z)=\int_{0}^{\infty} \frac{\lambda \beta t^{\beta-1} \mathrm{e}^{-\lambda t}}{\Gamma(\beta)} \mathrm{e}^{-z t} \mathrm{~d} t=\int_{0}^{\infty} \frac{\lambda \beta t^{\beta-1} \mathrm{e}^{-(\lambda+z) t}}{\Gamma(\beta)} \mathrm{d} t=\frac{\lambda \beta}{\Gamma(\beta)} \int_{0}^{\infty} t^{\beta-1} \mathrm{e}^{-(\lambda+z) t} \mathrm{~d} t .
$$

Set $G$, the function defined by $G(z)=\int_{0}^{\infty} t^{\alpha} \mathrm{e}^{-z t} \mathrm{~d} t$. Using the change of variable $u=z t ; G(z)$ becomes, $G(z)=\int_{0}^{\infty} \frac{u^{\alpha}}{z^{\alpha+1}} \mathrm{e}^{-u} \mathrm{~d} u$; and this gives, $G(z)=\frac{1}{z^{\alpha+1}} \int_{0}^{\infty} u^{\alpha} \mathrm{e}^{-u} \mathrm{~d} u$

Therefore, $G(z)=\int_{0}^{\infty} t^{\alpha} \mathrm{e}^{-z t} \mathrm{~d} t=\frac{\Gamma(\alpha+1)}{z^{\alpha+1}}$. Replacing $\alpha$ by $(\beta-1)$ and $z$ by $(z+\lambda)$, one gets, $\int_{0}^{\infty} t^{\beta-1} \mathrm{e}^{-(z+\lambda) t} \mathrm{~d} t=\frac{\Gamma(\beta)}{(\lambda+z)^{\beta}}$. Thus, $F(z)=\frac{\lambda \beta}{\Gamma(\beta)} \times \frac{\Gamma(\beta)}{(\lambda+z)^{\beta}}$, $\forall z \neq-\lambda$. Hence, $F(z)=\frac{\lambda \beta}{(\lambda+z)^{\beta}}, \forall z \neq-\lambda \quad$ (e.g. see [13] and [14]). -

\subsection{Generalized Gamma Distribution and Its Laplace Transform}

Proposition 6. The Laplace transform for a generalized distribution with parameters $\lambda, \beta, \alpha$ and density function $f(x)=\frac{\alpha \lambda \beta x^{\alpha \beta-1} \mathrm{e}^{-\lambda \alpha x}}{\Gamma(\beta)}$ is

$$
F(z)=\frac{\alpha \lambda \beta \Gamma(\alpha \beta)}{\Gamma(\beta)(\lambda \alpha+z)^{\beta}}
$$

with $z \neq-\lambda \alpha$.

\section{Proof of Proposition 6:}

Again the Laplace is defined as, $F(z)=\int_{0}^{\infty} f(t) \mathrm{e}^{-z t} \mathrm{~d} t$. It comes that, $F(z)=\int_{0}^{\infty} \frac{\alpha \lambda \beta t^{\alpha \beta-1} \mathrm{e}^{-\lambda \alpha t}}{\Gamma(\beta)} \mathrm{e}^{-z t} \mathrm{~d} t$ which can be rewritten as $F(z)=\int_{0}^{\infty} \frac{\alpha \lambda \beta t^{\alpha \beta-1} \mathrm{e}^{-(\lambda \alpha+z) t}}{\Gamma(\beta)} \mathrm{d} t=\frac{\alpha \lambda \beta}{\Gamma(\beta)} \int_{0}^{\infty} t^{\alpha \beta-1} \mathrm{e}^{-(\alpha \lambda+z) t} \mathrm{~d} t$. Replacing $\alpha$ by $(\alpha \beta-1)$ and $z$ by $(z+\alpha \lambda)$, one gets, $\int_{0}^{\infty} t^{\alpha \beta-1} \mathrm{e}^{-(z+\alpha \lambda) t} \mathrm{~d} t=\frac{\Gamma(\alpha \beta)}{(\alpha \lambda+z)^{\alpha \beta}}$. Therefore, $F(z)=\frac{\alpha \lambda \beta}{\Gamma(\beta)} \times \frac{\Gamma(\alpha \beta)}{(\alpha \lambda+z)^{\alpha \beta}}$ if $z \neq-\lambda \alpha$. Hence, $F(z)=\frac{\alpha \lambda \beta \Gamma(\alpha \beta)}{\Gamma(\beta)(\lambda \alpha+z)^{\beta}}$, provided $\mathrm{z} \neq-\lambda \alpha$.

\subsection{Pareto Distribution and Its Laplace Transform}

Proposition 7. The Laplace transform of the Pareto distribution with parameters $\lambda, \theta$ and density function $f_{x}(x)=\frac{\theta \lambda^{\theta}}{x^{\theta+1}}$ with $x \geq \lambda$ and $\lambda, \alpha$ positive; is given by, 


$$
F(z)=\theta \lambda^{\theta} z^{\theta}[\Gamma(-\theta)-I(z \lambda,-\theta) \Gamma(-\theta)]
$$

where $\Gamma$ is defined as before, whereas $I$ is given by, $I(t, \alpha)=\frac{1}{\Gamma(\alpha)} \times \int_{0}^{t} x^{\alpha-1} \mathrm{e}^{-x} \mathrm{~d} x$.

Proof of Proposition 7:

The Laplace transform is defined as, $F(z)=\int_{0}^{\infty} f(t) \mathrm{e}^{-z t} \mathrm{~d} t$, so that $F(z)=\int_{\lambda}^{\infty} \frac{\theta \lambda^{\theta}}{t^{\theta+1}} \mathrm{e}^{-z t} \mathrm{~d} t$. Using the change of variable $u=z t$, one gets $F(z)=\int_{z \lambda}^{\infty} \frac{\theta \lambda^{\theta}}{z u^{\theta+1}} \mathrm{e}^{-u} \mathrm{~d} u=\theta \lambda^{\theta+1} z^{\theta} \int_{z \lambda}^{\infty} u^{-\theta-1} \mathrm{e}^{-u} \mathrm{~d} u$ which leads to, $F(z)=\theta \lambda^{\theta} z^{\theta}\left(\int_{0}^{\infty} u^{-\theta-1} \mathrm{e}^{-u} \mathrm{~d} u-\int_{0}^{z \lambda} u^{-\theta-1} \mathrm{e}^{-u} \mathrm{~d} u\right)$.

Thus, $F(z)=\theta \lambda^{\theta} z^{\theta}[\Gamma(-\theta)-I(z \lambda,-\theta) \Gamma(-\theta)]$ where $I$ is defined as: $I(t, \alpha)=\frac{1}{\Gamma(\alpha)} \times \int_{0}^{t} x^{\alpha-1} \mathrm{e}^{-x} \mathrm{~d} x$

\subsection{Log-Normal Distribution and Its Laplace Transform}

Proposition 8. The Laplace transform of the log-normal distribution with parameters $\sigma$ and $\mu$ with density function $f_{X}(x)=\frac{\exp \left(-\frac{1}{2}\left(\frac{\ln x-\mu}{\sigma}\right)^{2}\right)}{x \sqrt{2 \pi} \sigma}$ with $x$ positive; is given by:

$$
F(z)=E_{X}\left(\mathrm{e}^{-z \mathrm{e}^{X}}\right)
$$

where $E_{X}$ is the mathematical expectation of a normal random distribution truncated at 0 and with parameters $\sigma$ and $\mu$.

\section{Proof of Proposition 8:}

The Laplace is by definition, $F(z)=\int_{0}^{\infty} f(t) \mathrm{e}^{-z t} \mathrm{~d} t$, so that $F(z)=\int_{0}^{\infty} \frac{\exp \left(-\frac{1}{2}\left(\frac{\ln t-\mu}{\sigma}\right)^{2}\right)}{t \sqrt{2 \pi} \sigma} \mathrm{e}^{-z t} \mathrm{~d} t$. By setting $x=\ln t$, one obtains, $F(z)=\int_{0}^{\infty} \frac{\exp \left(-\frac{1}{2}\left(\frac{x-\mu}{\sigma}\right)^{2}\right)}{\mathrm{e}^{x} \sqrt{2 \pi} \sigma} \mathrm{e}^{-z \mathrm{e}^{x}} \mathrm{e}^{x} \mathrm{~d} x=\int_{0}^{\infty} \frac{\exp \left(-\frac{1}{2}\left(\frac{x-\mu}{\sigma}\right)^{2}\right)}{\sqrt{2 \pi} \sigma} \mathrm{e}^{-z \mathrm{e}^{x}} \mathrm{~d} x=E_{X}\left(\mathrm{e}^{-z \mathrm{e}^{x}}\right)$ where $E_{X}$ is the mathematical expectation of a normal random distribution truncated below at 0 and with parameters $\sigma$ and $\mu$.

\subsection{Log-Logistic Distribution and Its Laplace Transform}

Proposition 9. The Laplace transform of the log-logistic distribution with pa- 
rameters $\lambda, \alpha$ and with density function: $f_{X}(x)=\frac{\alpha x^{\alpha-1} \lambda}{\left(1+\lambda x^{\alpha}\right)^{2}}$ with $\lambda, \alpha$ positive; is:

$$
F(z)=E_{X}\left(\mathrm{e}^{-z X}\right)
$$

where $E_{X}$ is the mathematical expectation of a log-logistic random distribution with parameters $\alpha$ and $\lambda$.

\section{Proof of Proposition 9:}

The Laplace transform is by definition, $F(z)=\int_{0}^{\infty} f(t) \mathrm{e}^{-z t} \mathrm{~d} t$. Note that the function defined by, $g_{X}(x)=\frac{\alpha x^{\alpha-1} \lambda}{\left(1+\lambda x^{\alpha}\right)^{2}} \mathrm{e}^{-z x}$ is continuously differentiable on the interval admitting a limit equal to zero in the neighbourhood of $+\infty$. The Laplace transform therefore always exits. Thus, $F(z)=\int_{0}^{\infty} \frac{\alpha \lambda t^{\alpha-1}}{\left(1+\lambda t^{\alpha}\right)^{2}} \mathrm{e}^{-z t} \mathrm{~d} t$. Hence, $F(z)=E_{X}\left(\mathrm{e}^{-z X}\right)$ where $E_{X}$ is the mathematical expectation of a log-logistic random distribution with parameters $\alpha$ and $\lambda$.

\subsection{Gompertz Distribution and Its Laplace Transform}

Proposition 10. The Laplace transform of the Gompertz distribution with parameters $\theta$ and $\alpha$ with density function: $f_{X}(x)=\theta \mathrm{e}^{\alpha x} \exp \left[\frac{\theta}{\alpha}\left(1-\mathrm{e}^{\alpha x}\right)\right]$ with $\theta, \alpha$ positive is:

$$
F(z)=\int_{0}^{\infty} \theta \mathrm{e}^{(\alpha-z) t} \exp \left[\frac{\theta}{\alpha}\left(1-\mathrm{e}^{\alpha t}\right)\right] \mathrm{d} t
$$

\section{Proof of Proposition 10:}

The Laplace is, $F(z)=\int_{0}^{\infty} f(t) \mathrm{e}^{-z t} \mathrm{~d} t$. Thus,

$$
F(z)=\int_{0}^{\infty} \theta \mathrm{e}^{\alpha t} \exp \left[\frac{\theta}{\alpha}\left(1-\mathrm{e}^{\alpha t}\right)\right] \mathrm{e}^{-z t} \mathrm{~d} t=\int_{0}^{\infty} \theta \mathrm{e}^{(\alpha-z) t} \exp \left[\frac{\theta}{\alpha}\left(1-\mathrm{e}^{\alpha t}\right)\right] \mathrm{d} t \quad \text { (e.g. } \quad \text { see }
$$

[14] and [15]).

\section{Conclusion}

In this paper one derived the Laplace transform of some important distributions used in counting processes. Results have important implications in 1) solving differential equations; 2) solving partial derivative equations; 3) deriving complex impedances; 4) solving partial fraction expansions; 5) conducting convolution analysis; 6) running complex geometric analyses; 7) determining structure of astronomical objects etc.

\section{Conflicts of Interest}

The authors declare no conflicts of interest regarding the publication of this paper. 


\section{References}

[1] Garcia, J.M.A. (2005) Explicit Solutions for Survival Probabilities in the Classical Risk Model. ASTIN Bulletin, 35, 113-130. https://doi.org/10.1017/S0515036100014082

[2] Bhullar, M.S. (2018) Study on Properties and Applications of Laplace Transformation: A Review. Pramana Research Journal, 8, 246-251.

[3] Bracewell, R.N. (2000) The Fourier Transform and Its Applications. 3rd Edition, McGraw-Hill, Boston, MA.

[4] Davies, B. (2002) Integral Transforms and Their Applications. 3rd Edition, Springer, New York.

[5] Dyke, P.P.G. (2001) An Introduction to Laplace Transforms and Fourier Series. Springer, London. https://doi.org/10.1007/978-1-4471-0505-3

[6] Feller, W. (1971) An Introduction to Probability Theory and Its Applications. Vol. 2, 2nd Edition, John Wiley and Sons, New York.

[7] Higgins, J.J. and Keller-McNulty, S. (1995) Concepts in Probability and Stochastic Modeling. Wadsworth Publishing Company, Belmont, CA.

[8] Jerri, A.J. (1985) Introduction to Integral Equations with Applications. Marcel Dekker, New York.

[9] Kalbfleisch, J.D. and Prentice, R.L. (2006) The Statistical Analysis of Failure Time Data. Wiley Series in Probability and Statistics.

[10] Marsden, J.E. and Hoffman, M.J. (1999) Basic Complex Analysis. WH Freeman, New York.

[11] Ortigueira, M.D., Torres, D.F.M. and Trujillo, J.J. (2016) Exponentials and Laplace Transforms on Non-Uniform Time Scales. Communications in Nonlinear Science and Numerical Simulation, 39, 252-270. https://doi.org/10.1016/j.cnsns.2016.03.010

[12] Parashar, B.P. (2008) Differential and Integral Equations. CBS Publication, New Delhi.

[13] Reddy, K.J.P. and Vaithyasubramanian, S. (2018) A Survey of Laplace Transform Applications in Various Fields of Science and Engineering. International Journal of Pure and Applied Mathematics, 119, 769-777.

[14] Ross, S.M. (2007) Introduction to Probability Models. 9th Edition, Academic Press, New York.

[15] Silverman, R.A. (1974) Complex Analysis with Applications. Prentice Hall, New York. 